\title{
FGFR2 alteration as a potential therapeutic target in poorly cohesive gastric carcinoma
}

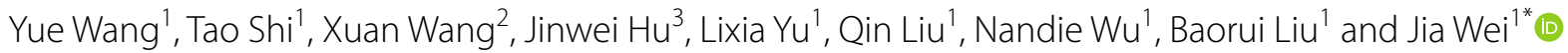

\begin{abstract}
Background: Poorly cohesive (PC) is a unique histologic subtype of gastric cancer (GC), with an increasing incidence in recent years. However, the molecular characteristics and therapeutic targets of PC GC are not yet well studied and there are no effective therapies for these patients.

Methods: Formalin fixed paraffin embedded (FFPE) samples of 556 GC patients, including 64 PC GC, were collected for next-generation sequencing (NGS). Clinical characteristics and genomic profiling were analyzed. FGFR2 expression was detected by quantitative real time polymerase chain reaction (qRT-PCR) and immunohistochemistry (IHC). FGFR2 inhibitors response was studied in vitro.

Results: Among $556 \mathrm{GC}$ patients, PC GC patients were younger ( $\mathrm{P}=0.004)$, had lower tumor mutation burden (TMB$\mathrm{L})(\mathrm{P}=0.001)$ than non-PC GC. The top 10 most frequently mutated genes in PC GC were TP53 (48\%), CDH1 (31\%), ARID1A (14\%), FGFR2 (14\%), ERBB2 (9\%), CDKN2A (9\%), FGF3 (8\%), LRP1B (9\%), FGF19 (8\%) and FGF4 (8\%). Noticeably, FGFR2 is more frequently mutated than non-PC GC (14\% vs. $6 \%, P=0.037)$, including copy number variants (CNVs, 12.5\%) and gene rearrangements (3.1\%, FGFR2NTI1 A and FGFR2/TACC2). Former studies have confirmed that gain of copy number could increase FGFR2 expression and sensitivity to FGFR2 inhibitors in GC. However, no research has verified the function of FGFR2 rearrangements in GC. Our results showed that cell lines of GC transfected with TACC2FGFR2 fusion had increased mRNA and protein expression of FGFR2, and were more sensitive to FGFR2 inhibitors. FGFR2 inhibitors might be a new therapeutic target for PC GC. In addition, we found patients of PC GC harboring gene rearrangements $(n=9)$ had poorer overall survival $(O S)$ in comparison with patients without any gene rearrangement $(n=19)(16.0$ months vs 21.0 months, $P=0.043)$. Gene rearrangement might be an adverse prognostic factor for PC GC patients.
\end{abstract}

Conclusions: FGFR2 alterations were recurrent in PC GC and FGFR2 inhibitors might be a new therapeutic target for PC GC.

Keywords: Gastric cancer, Poorly cohesive, Next-generation sequencing, Gene rearrangement, Fibroblast growth factor receptors 2

*Correspondence: jiawei99@nju.edu.cn

${ }^{1}$ The Comprehensive Cancer Centre of Nanjing Drum Tower Hospital, The Affiliated Hospital of Nanjing University Medical School \& Clinical Cancer Institute of Nanjing University, No. 321, Zhongshan Road, Nanjing 210008, China

Full list of author information is available at the end of the article

\section{Background}

Gastric cancer (GC) is the fifth most common cancer and the third leading cause of cancer mortality in the word [1]. Numerous studies have shown that GC is histologically and genetically heterogeneous. The WHO classified GC into papillary adenocarcinoma, tubular adenocarcinoma, mucinous adenocarcinoma, poorly cohesive (PC) carcinoma, mixed adenocarcinoma and other rare subtypes [2]. In recent years, a decrease occurred in the original author(s) and the source, provide a link to the Creative Commons licence, and indicate if changes were made. The images or other third party material in this article are included in the article's Creative Commons licence, unless indicated otherwise in a credit line to the material. If material is not included in the article's Creative Commons licence and your intended use is not permitted by statutory regulation or exceeds the permitted use, you will need to obtain permission directly from the copyright holder. To view a copy of this licence, visit http://creativecommons.org/licenses/by/4.0/. The Creative Commons Public Domain Dedication waiver (http://creativeco mmons.org/publicdomain/zero/1.0/) applies to the data made available in this article, unless otherwise stated in a credit line to the data. 
overall incidence of $\mathrm{GC}$, however, the proportion of GC with WHO PC histology is increasing [3]. PC GC is defined as tumor composed of isolated or small groups of tumor cells by the WHO classification [3]. Compared to other GC subtypes, PC GC is poorly differentiated and easy to metastasized. None of current treatments, including chemotherapy, radiotherapy, targeted therapy and immunotherapy, has shown good results $[4,5]$.

Although the histopathological classifications currently remain the most commonly used for therapy decision making in the clinical setting, molecular classifications have been developed to guide future treatment development [5]. Several different molecular GC classification systems have been proposed these years, attempting to relate molecular features to histological phenotypes and clinical features [6, 7]. In 2014, The Cancer Genome Atlas (TCGA) research network proposed four molecularly distinct GC subtypes: Epstein-Barr virus infected (EBV), microsatellite instability (MSI), genomically stable (GS), and chromosomal instability (CIN) [8]. Also, in 2015, the Asian Cancer Research Group classified GC into four molecular subtypes: mesenchymal-like, microsatelliteunstable, the tumor protein 53 (TP53)-active and TP53inactive types [9]. However, there are few detailed studies on the mutational spectrum of PC GC. Further investigation of the genetic alterations may provide useful information to explore new therapies for PC GC patients.

In this study, we compared the clinical characteristics and genomic profiling of PC GC patients and non-PC GC patients. Furthermore, we investigated whether genetic alterations are associated with patient prognosis in PC GC. Finally, we tried to seek new therapies according to specific genetic alterations of PC GC.

\section{Methods}

\section{Patients and samples}

We collected 556 patient samples of gastric adenocarcinoma. All histopathologic diagnoses were reviewed by at least two senior pathologists independently. Clinical information was retrospectively collected and the overall survival (OS) was measured from the date of surgery to the date of death or the last follow-up visit. The last follow-up date was August 8th, 2016. This study was conducted in accordance with the code of ethics of the World Medical Association (Declaration of Helsinki) and approved by the Ethics Committee of Nanjing Drum Tower Hospital (No. 2016-196-01).

Nucleic acid preparations and next-generation sequencing Formalin fixed paraffin-embedded (FFPE) tissues obtained from patients with GC were collected for NGS in a 450-gene panel assay, whose accuracy has been verified before (Additional file 1: Figure S1). DNA was extracted from the unstained FFPE sections with tumor content of no less than $20 \%$ and was fragmented to $\sim 250$ bp by sonication. A library was constructed using the KAPA Hyper Prep Kit (KAPA Biosystems) and hybridization capture was performed with a custom panel containing individually synthesized 5'-biotinylated DNA probes. The capture probes target exons of 450 cancer-related genes and frequently rearranged introns of 38 genes. Paired-end sequencing was performed according to the manufacturer's protocols. Genomic alterations, including single base substitutions, short and long insertions/deletions (INDELs), copy number variations, and gene rearrangements, were assessed using the OrigiMedpipeline. Genomic alterations relevant for cancer immunotherapy, which included TMB levels and MSI, were also evaluated $[10,11]$. Mutation signature was predicted using a public software deconstuctSigs. All substitution mutations were classified into 96 kinds of trinucleotides and the frequency of each was precisely calculated as the characteristic signature of these samples. The signature was then compared with the typical 30 signatures from COSMIC to identify the most similar combination and the percentage of each contributed [12].

\section{Cell lines}

To verify the function of TACC2-FGFR2 fusion, we generated gastric cancer cell lines with stable expression of TACC2-FGFR2. The Lenti-EF1a-EGFP-pGK-Puro retroviral vectors containing the particular fusion genes were transfected into $293 \mathrm{~T}$ cells to produce virus. MKN45 and NUGC4 cells were then infected with the viral supernatant containing expression constructs. Stable transfectants were obtained and maintained under selection pressure by puromycin dihydrochloride $(2 \mu \mathrm{g} / \mathrm{ml}$, Beyotime, ST551-10 mg). Under selection conditions, clones were picked and maintained.

\section{Quantitative real time polymerase chain reaction (qRT-PCR) analysis}

Total RNAs were extracted by TRIzol ${ }^{\mathrm{TM}}$ Reagent (Thermo Fisher Scientific, 15596018), then dissolved in RNasefree water. cDNA synthesis is performed in the first step using total RNA, Random Primers (Thermo Fisher Scientific, 48190011) and dNTP Mix (10 mM ea, 18427013), $5 \mathrm{~min}$ at $65^{\circ} \mathrm{C}$. In the second step, PCR is performed in a separate tube using probes specific for the gene of interest, M-MLV Reverse Transcription (200 U/ul, 28025013) and RNase OUT ${ }^{\mathrm{TM}}$ Recombinant Ribonuclease Inhibitor (Thermo Fisher Scientific, 10777019), following the procedures of $10 \mathrm{~min}$ at $25^{\circ} \mathrm{C}, 45 \mathrm{~min}$ at $37^{\circ} \mathrm{C}, 10 \mathrm{~min}$ at $70{ }^{\circ} \mathrm{C}$, and then $4{ }^{\circ} \mathrm{C}$. TaqMan ${ }^{\mathrm{TM}}$ Fast Advanced Master Mix (Thermo Fisher Scientific, 444556) was used with the following PCR parameters, 1 cycle of $20 \mathrm{~s}$ at 
$95{ }^{\circ} \mathrm{C}, 40$ cycles of $1 \mathrm{~s}$ at $95^{\circ} \mathrm{C}$ and $20 \mathrm{~s}$ at $60{ }^{\circ} \mathrm{C}$ using QuantStudio $^{\text {тм }} 7$ Flex (Applied Biosystems). Probes used in this study are FGFR2 (Thermo Fisher Scientific, Hs01552918_m1) and $\beta$-actin (Thermo Fisher Scientific, Hs99999903_m1).

\section{Immunohistochemical staining of tumor tissues}

The expression of PD-1, PD-L1, CD3, FGFR2 in tumors was evaluated via immunohistochemical analysis (antiPD-1, 1:100, NAT105, Cell Marque; anti-PD-L1, 1:100, SP142, Spring Bio; anti-CD3, 1:500, CD3-565-L-CE, Leica/Novocastra; anti-FGFR2, 1:200, ab58201, Abcam). The PD-1, PD-L1 is observable in the cytoplasm or on the membrane of the tumor cell or the TILs. The immunoreactivity of PD-1, PD-L1 was evaluated semiquantitatively according to the percentage and intensity of positive cells. Specimens in which PD-1 or PD-L1 were observed in more than $1 \%$ of tumor cells or immune cells were considered PD-1 or PD-L1 positive. CD3 was detected in the nuclei of the TILs. The distribution of CD3+ TILs was observed in the areas with the highest density of TILs first at low magnification. The amount of positive TILs was then recorded at high magnification (HPF $400 \times$ magnification). The number of CD3+ TILs was determined in 30 random high power fields in each section.

\section{Fluorescence in situ hybridization (FISH)}

Two-color FISH was performed on $2 \mu \mathrm{m}$ thick sections from formalin-fixed, paraffin-embedded tumor tissues with FGFR2 rearrangements and paired normal tissues. Before hybridization, sections were deparaffinized, dehydrated in $100 \%$ ethanol, and air-dried. Commercially available, locus-specific FGFR2 probe (anbiping, F.0119701) were used according to the manufacturer's recommendations. 377-kb Spectrum Green directly labeled fluorescent DNA in the $3^{\prime}$ of FGFR2 and 446-kb Spectrum Red directly labeled fluorescent DNA in the $5^{\prime}$ of FGFR2.

\section{Drug sensitivity test}

Cell counting kit-8 (CCK-8) (Vazyme, A311-01/02) assay was used to estimate drug response. Briefly, 2500 cells were seeded each well of 96-well plates with $100 \mu \mathrm{l}$ of 10\% FBS 1640 medium, and treated with BGJ398 (Selleck, S2183), AZD4547 (Selleck, S2801) or Erdafitinib (Selleck, S8401) on the 2nd day. After additional days of incubation, $10 \mu \mathrm{l}$ CCK- 8 was added into each well and incubated for $2 \mathrm{~h}$. Afterwards, absorbance was measured at $450 \mathrm{~nm}$ with microplate reader. The IC50 values were calculated with nonlinear regression analysis by using GraphPad.

\section{Statistical analysis}

Statistical analysis was performed by SPSS statistics software, version 25.0 (SPSS, Chicago, IL, USA). The continuous variables were tested for normal distribution before analyzing by $\mathrm{t}$ test. The categorical variables were taken with the Pearson's Chi-square test (or Fisher's exact test). Impact of clinical characteristics and genetic alterations on survival outcomes were estimated by using KaplanMeier method, Cox proportional hazard modeling. A two-tailed P value of less than 0.05 was regarded as statistically significant.

\section{Results \\ Clinical characteristics and genomic profiling of PC GC and non-PC GC patients}

In this study, a total of 556 GC samples were included. Among all GC patients, 64 (11.5\%) are diagnosed as PC GC and 492 (88.5\%) are non-PC GC. We compared the clinical characteristics between PC GC and non-PC GC patients (Table 1). It showed that PC GC patients were younger than non-PC GC (proportion of patients younger than 60 years old was $65.6 \%$ vs. $46.3 \%, P=0.004$ ) (Table 1). Also, PC GC patients are more likely to be female, although there was no significant difference (proportion of female patients was $40.6 \%$ vs. $32.7 \%, P=0.208$ ) (Table 1).

Formalin fixed paraffin-embedded (FFPE) tissues obtained from these 556 GC patients were collected for NGS in a 450-gene panel assay (Fig. 1a). Microsatellite instability (MSI) status was identified based on mismatch repair (MMR) gene expression, while tumor mutation burden (TMB) was defined as mutations per megabase (Muts/Mb). NGS analysis revealed all 64 gastric PC GC patients were MSS, while 29 (5.9\%) non-PC GC patients were MSI-H $(P=0.085)$ (Table 1$)$. Similarly, only $9.4 \%$ (6/64) PC GC patients had high TMB ( $\geq 10$ Muts/Mb) while the proportion among non-PC GC patients was 28.3\% (139/492) $(P=0.001)$ (Table 1).

The top 10 most frequently mutated genes among 64 gastric PC GC patients were TP53 (48\%), CDH1 (31\%), ARID1A (14\%), FGFR2 (14\%), CDKN2A (9\%), ERBB2 (9\%), LRP1B (9\%), FGF19 (8\%), FGF3 (8\%), and FGF4 (8\%), compared to TP53 (66\%), ARID1A (20\%), LRP1B (16\%), CDH1 (14\%), PIK3CA (12\%), FAT4 (10\%), TGFBR2 (10\%), KRAS (10\%), APC (9\%) and KMT2D (8\%) in non-PC GC patients (Fig. 1a). Genomic alterations including single nucleotide variants (SNVs), INDELs, copy number variations (CNVs), gene fusions and rearrangements were assessed. We respectively compared the most frequent SNVs and INDELs, CNVs and rearrangements between PC GC and non-PC GC. SNVs and INDELs of TP53 (64.8\% vs $46.9 \%, P=0.005)$, CNVs 
Table 1 Clinical characteristics and genomic profiling of PC GC and non-PC GC patients $(N=556)$

\begin{tabular}{|c|c|c|c|c|}
\hline & No. of patients & $\begin{array}{l}P C \\
n=64(\%)\end{array}$ & $\begin{array}{l}\text { Non-PC } \\
n=492(\%)\end{array}$ & $P^{b}$ \\
\hline Age (years) & 556 & & & 0.004 \\
\hline$<60$ & 270 & $42(65.6 \%)$ & $228(46.3 \%)$ & \\
\hline$\geq 60$ & 286 & $22(34.4 \%)$ & $264(53.7 \%)$ & \\
\hline Gender & 556 & & & 0.208 \\
\hline Male & 369 & $38(59.4 \%)$ & $331(67.3 \%)$ & \\
\hline Female & 187 & $26(40.6 \%)$ & $161(32.7 \%)$ & \\
\hline MSI status & 545 & & & 0.085 \\
\hline MSS & 516 & $64(100 \%)$ & $452(91.8 \%)$ & \\
\hline MSI-H & 29 & $0(0 \%)$ & $29(5.9 \%)$ & \\
\hline $\mathrm{TMB}^{\mathrm{a}}$ & 556 & & & 0.001 \\
\hline TMB-L & 411 & $58(90.6 \%)$ & $353(71.7 \%)$ & \\
\hline TMB-H & 145 & $6(9.4 \%)$ & $139(28.3 \%)$ & \\
\hline \multicolumn{5}{|c|}{ Gene SNVs and INDELs } \\
\hline TP53 & 349 & $30(46.9 \%)$ & $319(64.8 \%)$ & 0.005 \\
\hline $\mathrm{CDH} 1$ & 80 & $18(28.1 \%)$ & $62(12.6 \%)$ & 0.001 \\
\hline ARID1A & 98 & $9(14 \%)$ & $89(18.1 \%)$ & 0.426 \\
\hline ERBB2 & 29 & $6(9.4 \%)$ & $23(4.7 \%)$ & 0.196 \\
\hline CDKN2A & 17 & $4(6.3 \%)$ & $13(2.6 \%)$ & 0.234 \\
\hline $\mathrm{RHOA}$ & 32 & $4(6.3 \%)$ & $28(5.7 \%)$ & 1.000 \\
\hline SMAD4 & 33 & $2(3.1 \%)$ & $31(6.3 \%)$ & 0.465 \\
\hline \multicolumn{5}{|l|}{ Gene CNVs } \\
\hline FGFR2 & 33 & $8(12.5 \%)$ & $25(5.1 \%)$ & 0.037 \\
\hline CCNE1 & 63 & $2(3.1 \%)$ & $61(12.4 \%)$ & 0.028 \\
\hline ERBB2 & 42 & $1(1.6 \%)$ & $41(8.3 \%)$ & 0.094 \\
\hline VEGFA & 28 & $1(1.6 \%)$ & $27(5.5 \%)$ & 0.295 \\
\hline \multicolumn{5}{|c|}{ Gene rearrangements } \\
\hline FGFR2 & 8 & $2(3.1 \%)$ & $6(1.2 \%)$ & 0.518 \\
\hline
\end{tabular}

CNV copy number variations, INDEL short and long insertion/deletion, MSI microsatellite instability, MSI-H microsatellite instability-high, MSS microsatellite stable, $P C$ poorly cohesive, $S N V$ single nucleotide variant, $T M B$ tumor mutation burden, $T M B-H$ tumor mutational burden-high, $T M B-L$ tumor mutational burdenlow

a Tumors with $\mathrm{TMB}<10$ Muts/Mb are defined as TMB-L, while $\geq 10$ Muts/Mb defined as TMB-H

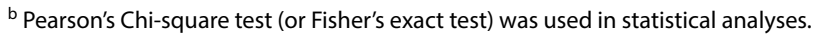
Values in italic are statistically significant

of CCNE1 (12.4\% vs $3.1 \%, P=0.028)$ occurred more frequently in non-PC GC while SNVs and INDELs of CDH1 (12.6\% vs $28.1 \%, P=0.001)$, CNVs of FGFR2 (5.1\% vs $12.5 \%, P=0.037)$ occurred more frequently in PC GC (Table 1).
The value of gene rearrangements in predicting prognosis for PC GC

We detected gene rearrangements in 20.3\% (13/64) PC GC patients (Fig. 1b). Specially, FGFR2 rearrangements (FGFR2/VTI1A and FGFR2/TACC2) were recurrently detected in 3.1\% (2/64) PC GC tumor samples. We collected clinical characteristics and prognostic information of 28 PC GC patients from Drum Tower Hospital. It demonstrated that PC GC patients with gene rearrangements had a higher $\mathrm{N}$ stage $(P=0.001)$ and a higher tumor stage $(P=0.010)$ defined by 8 th American Joint Committee on Cancer (AICC) criterion (Table 2). Moreover, patients harboring gene rearrangements $(n=9)$ had a shorter overall survival (OS) in comparison with patients without any gene rearrangement $(\mathrm{n}=19)$ (16.0 months vs 21.0 months, $P=0.043$ ) (Fig. 2a). To further explore the risk factors related to survival outcome of PC GC, we employed univariate and multivariate Cox regression analyses to identify protective or adverse prognostic factors. As shown in Additional file 1: Table S1, independent prognosis factors of PC GC identified in the univariate Cox regression are age over 60 years old $(\mathrm{HR}=2.630,95 \% \mathrm{CI} 1.104-6.268$, $P=0.029)$, higher tumor stage $(\mathrm{HR}=2.506,95 \% \mathrm{CI}$ 1.026-6.122, $P=0.044)$, higher $\mathrm{N}$ stage $(\mathrm{HR}=3.789,95 \%$ CI 1.507-9.527, $P=0.005)$ and overlapped tumor location $(\mathrm{HR}=4.543,95 \% \mathrm{CI} 1.089-18.948, P=0.038)$. Also, patients with gene rearrangements was adverse prognosis factor for PC GC patients, although without significant difference ( $\mathrm{HR}=2.384,95 \%$ CI $0.993-5.723, P=0.052)$. Results of multivariate Cox regression suggested that among PC GC patients, age over 60 years old $(\mathrm{HR}=3.083$, 95\% CI 1.114-8.531, $P=0.030$ ) were considered as adverse prognosis factor. In addition, Cox's regression model was separately used to estimate HR and 95\% CI in each subgroup (Fig. 2b). Among IIIA-B stage ( $\mathrm{HR}=2.64,95 \% \mathrm{CI}$ 1.03-6.78, $P=0.043)$, $\mathrm{PD}-\mathrm{L} 1$ positive $(\mathrm{HR}=4.74,95 \% \mathrm{CI}$ 1.04-21.63, $P=0.045)$ PC GC patients, gene rearrangement was a negative factor for overall survival (Fig. 2b).

\section{Function of TACC2-FGFR2 fusion in PC GC}

Fluorescence in situ hybridization (FISH) and RNA sequencing (RNA-seq) was performed in two samples with FGFR2 rearrangements (FGFR2/VTI1A and FGFR2/TACC2). FGFR2/VTI1A and FGFR2/TACC2 rearrangements could both be detected by FISH in corresponding patient's samples (Additional file 1: Figure

(See figure on next page.)

Fig. 1 a Overview of recurrent somatic genomic alterations in GC patients. The patient samples are shown on the x-axis. Information of mutation rate, MSI status, gender, and patient age are shown on the top of $y$-axis, followed by the key genetic alterations including significant mutated genes. Frequency of each alteration were illustrated on the left or right of the mutation heat plot. b Overview of gene rearrangements in 64 gastric PC GC patients. Each line connecting two genes represented one gene rearrangement, and each color represented one patient. In all, 13 gastric PC GC patients had gene rearrangements. Orange arrow indicated the patient who had two gene rearrangements and they overlapped each other 


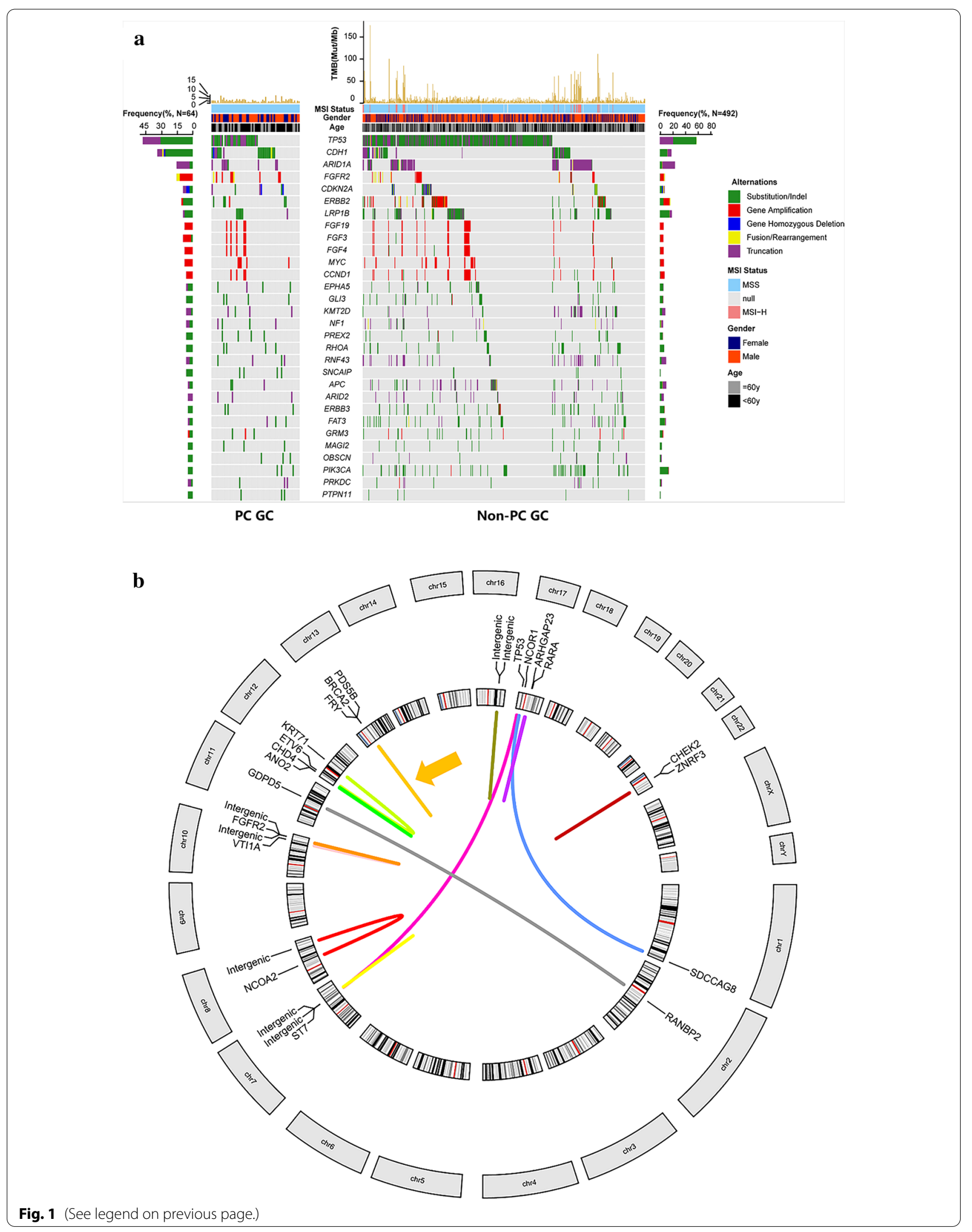


Table 2 Clinical characteristics of PC GC with and without gene rearrangements

\begin{tabular}{|c|c|c|c|c|}
\hline & $\begin{array}{l}\text { No. of } \\
\text { patients }\end{array}$ & $\begin{array}{l}\text { With } \\
\text { rearrangements } \\
n=9(\%)\end{array}$ & $\begin{array}{l}\text { Without } \\
\text { rearrangements } \\
n=19(\%)\end{array}$ & $P^{a}$ \\
\hline \multicolumn{4}{|l|}{ Age (years) } & \multirow[t]{3}{*}{0.371} \\
\hline$<60$ & 20 & $5(55.6 \%)$ & 15 (78.9\%) & \\
\hline$\geq 60$ & 8 & $4(44.4 \%)$ & $4(21.1 \%)$ & \\
\hline \multicolumn{4}{|l|}{ Gender } & \multirow[t]{3}{*}{0.249} \\
\hline Male & 17 & 7 (77.8\%) & $10(52.6 \%)$ & \\
\hline Female & 11 & $2(22.2 \%)$ & $9(47.4 \%)$ & \\
\hline \multicolumn{4}{|l|}{ AJCC } & \multirow[t]{3}{*}{0.010} \\
\hline$\| I \mid A-B$ & 17 & $2(22.2 \%)$ & 15 (78.9\%) & \\
\hline IIIC & 11 & $7(77.8 \%)$ & 4 (21.1\%) & \\
\hline \multicolumn{4}{|l|}{ T stage } & \multirow[t]{3}{*}{1.000} \\
\hline 3 & 17 & $5(55.6 \%)$ & 12 (63.2\%) & \\
\hline $4 a-4 b$ & 11 & $4(44.4 \%)$ & 7 (36.8\%) & \\
\hline \multicolumn{4}{|l|}{ N stage } & \multirow[t]{3}{*}{0.001} \\
\hline $1-3 a$ & 19 & $2(22.2 \%)$ & 17 (89.5\%) & \\
\hline $3 b$ & 9 & $7(77.8 \%)$ & $2(10.5 \%)$ & \\
\hline \multicolumn{5}{|c|}{ Tumor size (cm) } \\
\hline$\leq 4$ & 10 & $4(44.4 \%)$ & $6(31.6 \%)$ & \multirow[t]{2}{*}{0.677} \\
\hline$>4$ & 18 & $5(55.6 \%)$ & 13 (68.4\%) & \\
\hline \multicolumn{4}{|c|}{ Tumor location } & \multirow[t]{5}{*}{0.370} \\
\hline Upper & 7 & $4(44.4 \%)$ & $3(15.8 \%)$ & \\
\hline Middle & 7 & $1(11.1 \%)$ & $6(31.6 \%)$ & \\
\hline Lower & 10 & $3(33.3 \%)$ & $7(36.8 \%)$ & \\
\hline Overlap & 4 & $1(11.1 \%)$ & $3(15.8 \%)$ & \\
\hline \multicolumn{4}{|l|}{ PD-L1 } & \multirow[t]{3}{*}{0.689} \\
\hline Negative & 15 & $4(44.4 \%)$ & 11 (57.9\%) & \\
\hline Positive & 13 & $5(55.6 \%)$ & $8(42.1 \%)$ & \\
\hline \multicolumn{4}{|l|}{ PD-1 } & \multirow[t]{3}{*}{1.000} \\
\hline Negative & 22 & $7(77.8 \%)$ & 15 (78.9\%) & \\
\hline Positive & 6 & 2 (22.2\%) & 4 (21.1\%) & \\
\hline \multicolumn{4}{|l|}{ CD3 } & \multirow[t]{3}{*}{0.420} \\
\hline Low & 14 & $3(33.3 \%)$ & 11 (57.9\%) & \\
\hline High & 14 & $6(66.7 \%)$ & $8(42.1 \%)$ & \\
\hline
\end{tabular}

AJCC American Joint Committee on Cancer, $P C$ poorly cohesive

${ }^{a}$ Fisher's exact test was used in statistical analyses. Values in italic are statistically significant

S2). TACC2-FGFR2 fusion was verified in the form of TACC2 (exon1-2) -FGFR2 (exon5-18) by RNA-seq (Fig. 3a, Additional file 1: Figure S3). However, no RNA product of FGFR2-VTI1A rearrangement was detected by RNA-seq. We presumed that it might be due to no RNA product was transcribed by FGFR2-VTI1A rearrangement indeed. Next, we evaluated the expression level of FGFR2 protein in patient's surgical samples with TACC2-FGFR2 using immunohistochemistry (IHC), finding increased FGFR2 expression in tumor area compared to adjacent normal surface epithelial area (Fig. 3b).
To further investigate TACC2-FGFR2 fusion in PC GC, we stably expressed TACC2-FGFR2 in gastric cancer cell lines MKN45 and NUGC4. Using qRT-PCR, we found FGFR2 mRNA levels were increased in TACC2-FGFR2expressing MKN45 and NUGC4 cells (Fig. 4a). Moreover, IHC showed TACC2-FGFR2 upregulated FGFR2 protein expression in TACC2-FGFR2-expressing MKN45 and NUGC4 cells. (Fig. 4b).

To figure out the role of TACC2-FGFR2 fusion in targeted therapy for GC, we treated MKN45, NUGC4 cells and TACC2-FGFR2-expressing MKN45, NUGC4 cells with FGFR2 inhibitors, including BGJ398, AZD4547 and Erdafitinib. TACC2-FGFR2-expressing MKN45 and NUGC4 cells are more sensitive to all these three FGFR2 inhibitors (Fig. 4c).

\section{Discussion}

GC is a phenotypically and molecularly highly heterogeneous disease. Intratumoral, intrapatient and interpatient heterogeneity in GC remains a crucial barrier for targeted therapies [2]. PC GC is a unique subtype of GC, tending to metastasis and with poor prognosis $[13,14]$. None of current therapies showed satisfying results [4]. Therefore, there is a critical need to develop new efficacious therapeutic agents for PC GC. Molecular characteristics are becoming more and more important for GC treatment. Several molecular GC classification systems have been proposed these years for better clinical treatments $[8,9]$. A few papers have focused on genomic alterations of PC GC and presented significantly mutated genes identified are TP53, APC, KIT, EGFR, PIK3CA, CTNNB1, ARID1A, and CDH1 [13, 15-18]. Our results showed the top 10 most frequent altered genes among PC GC were TP53 (48\%), CDH1 (31\%), ARID1A (14\%), FGFR2 (14\%), ERBB2 (9\%), CDKN2A (9\%), FGF3 (8\%), LRP1B (9\%), FGF19 (8\%) and FGF4 (8\%), consistent with previous research.

However, these molecular characteristics of PC GC patients are still unclear for treatment and prognosis. To better understand the molecular characteristics of PC GC, we respectively analyzed SNVs and INDELs, $\mathrm{CNVs}$ and gene fusions and rearrangements. We found that 20\% (13/64) PC GC patients harboring somatic gene rearrangements. And, patients with rearrangements $(n=9)$ had a shorter overall survival (OS) in comparison with patients without any gene rearrangement $(\mathrm{n}=19)$ (16.0 months vs 21.0 months, $P=0.043$ ) (Fig. 3b). It may owe to gene rearrangements could form severe cancer driving structural variants (SV), including insertions, deletions, tandem duplications, inversions, translocations, and more complex rearrangements [19]. This is an indication that gene rearrangements play an important role in PC GC development and prognosis. Gene 
$\mathbf{a}$

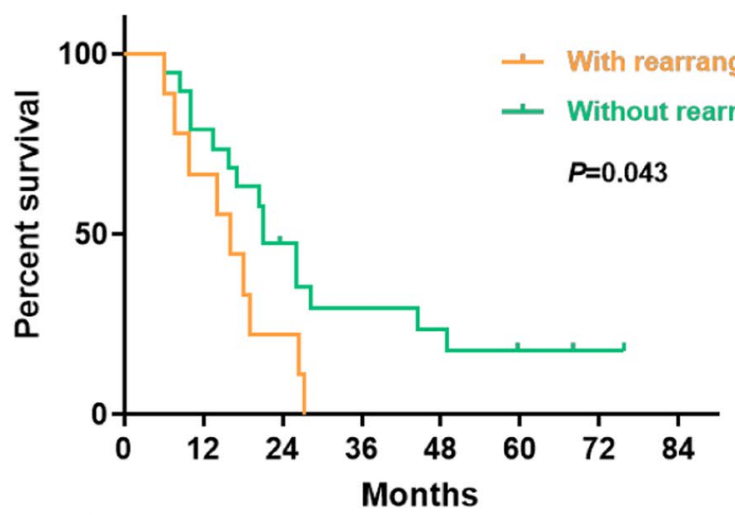

\begin{tabular}{r|lccccccc} 
No. at risk & \multicolumn{1}{|c}{ Time (mo) } \\
\hline With rearrangement & 9 & 6 & 2 & 0 & 0 & 0 & 0 & 0 \\
Without rearrangement & 19 & 15 & 8 & 5 & 4 & 2 & 1 & 0
\end{tabular}

b

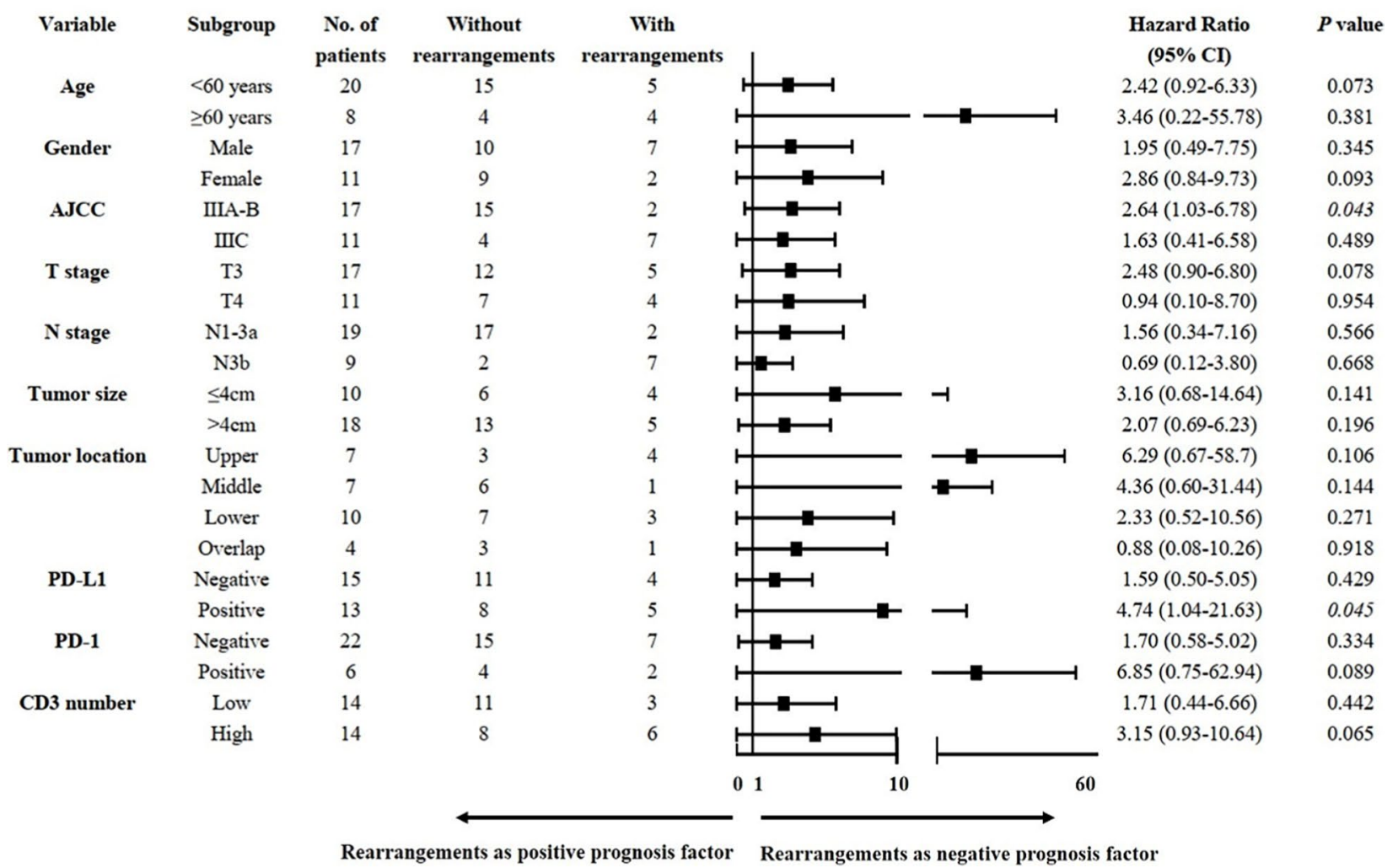

Fig. 2 a Kaplan-Meier estimates of survival probability. The survival curve of patients with and without rearrangements. $\mathbf{b}$ Subgroup analysis of PC GC patients with and without rearrangements

rearrangement might be an adverse prognostic factor for PC GC patients and more effective therapies targeting gene rearrangements should be studied for PC GC.

FGFR2, known as fibroblast growth factor receptor-2, is a transmembrane tyrosine kinase receptor, regulating cell proliferation, survival, migration and angiogenesis [20]. Genetic alterations in FGFR2, including gene amplification, mutations or rearrangements may dysregulate the FGF signaling pathway, influence the development and progression of various cancers by activating the 
a

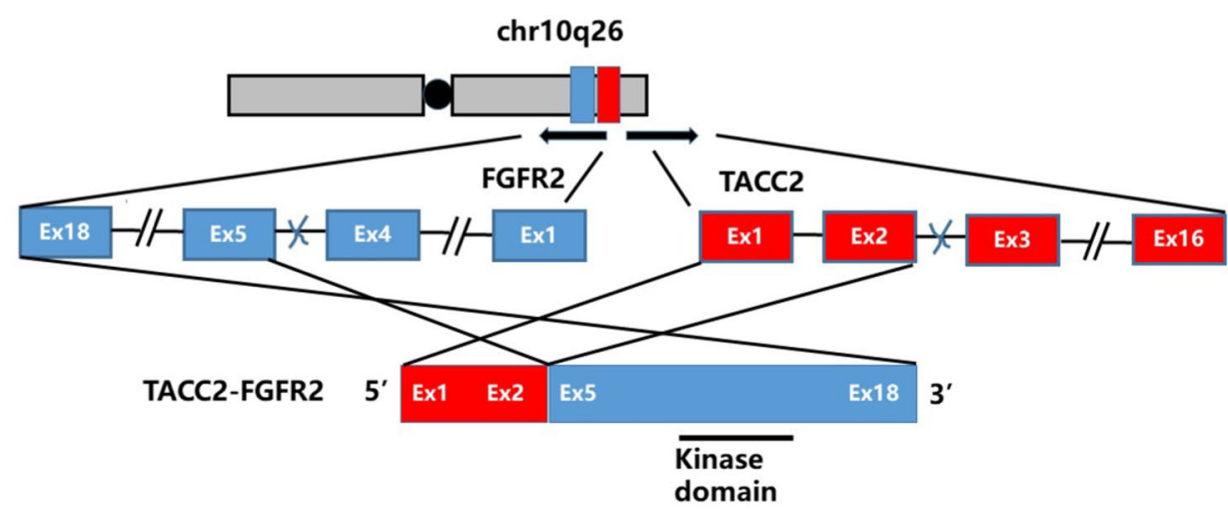

b

Tumor

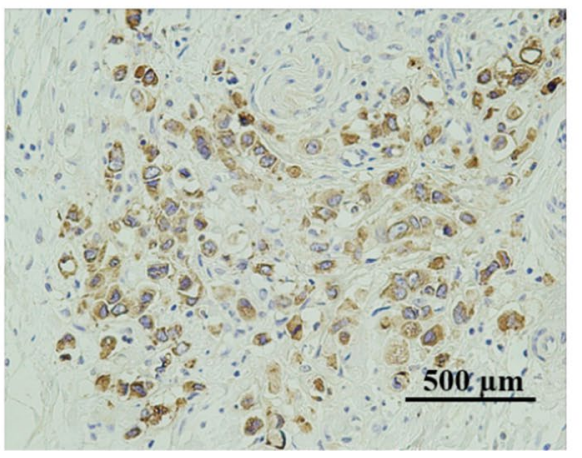

Normal

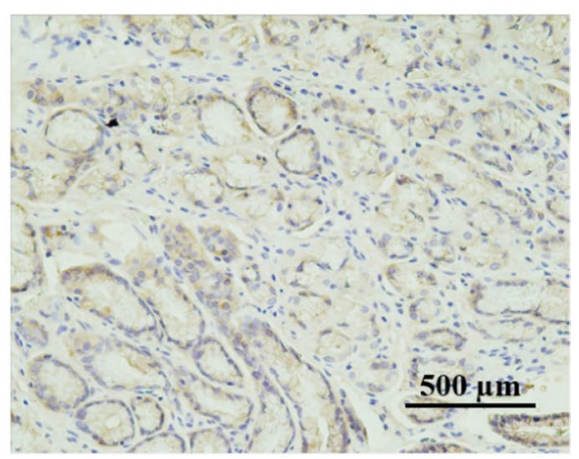

Fig. 3 a lllustration of breakpoint of TACC2 and FGFR2. Exons (Ex) of TACC2 and FGFR2 are indicated with red and blue, respectively. b Representative images of FGFR2 staining for PC GC patients with TACC2-FGFR2 rearrangement with tumor area (left), adjacent normal surface epithelial area (right). Scale bar $=500 \mu \mathrm{m}$

downstream PI3K-AKT and MAPK-ERK pathways [21]. Previous study demonstrated that FGFR2 overexpression, mainly due to FGFR2 gene amplification, is associated with poor pathological features, including deeper tumor invasion, more LN metastasis, advanced tumor stage, and worse survival in GC [22]. And, many researches indicated that the incidence of FGFR2 overexpression might differ between histological subtypes according to Lauren's classification [21]. Hattori found FGFR2 overexpression in $52.6 \%(20 / 38)$ diffuse-type GCs, but in none of 11 intestinal-type GCs [23]. PC GC tend to be diffuse-type according to Lauren's classification, however, no study verified the situation of FGFR2 genomic alterations in PC GC. Our results firstly showed that FGFR2 is more frequently mutated in PC GC than non-PC GC (14\% vs. $6 \%, \mathrm{P}=0.037$ ), including $12.5 \% \mathrm{CNVs}$. Several small-molecule inhibitors and antibodies for FGFR2 are under clinical trials [24, 25]. Recently, Catenacci [25] verified bemarituzumab, an IgG1 antibody specific for the FGFR2b receptor, seemed to be well tolerated and demonstrated single-agent activity as lateline therapy in patients with advanced-stage gastric and gastroesophageal junction adenocarcinoma (GEA). Moreover, bemarituzumab is currently being evaluated in combination with chemotherapy in a phase III trial as front-line therapy for patients with high FGFR2b-overexpressing advanced-stage GEA. Preliminary results showed that bemarituzumab, added to mFOLFOX6 chemotherapy, led to clinically meaningful and statistically significant improvements in PFS (9.5 m vs. $7.4 \mathrm{~m}, P=0.0727)$, OS (not reach vs. $12.9 \mathrm{~m}, P=0.0268)$ and ORR (47\% vs. 33\%). And, the higher the FGFR2 expression, the better the

(See figure on next page.)

Fig. 4 a qRT-PCR for the TACC2-FGFR2 fusion transcript in MKN45, NUGC4 cells and corresponding TACC2-FGFR2-expressing cells. Data are presented as mean \pm SEM. * means $P<0.05$, ** means $P<0.01$. b $\mathrm{IHC}$ of MKN45, NUGC4 cells and corresponding TACC2-FGFR2-expressing cells for FGFR2 protein. Scale bar $=50 \mu \mathrm{m}$. MKN45T:TACC2-FGFR2-expression MKN45 cells. NUGC4T: TACC2-FGFR2-expression NUGC4 cells. c Sensitivity of MKN45, NUGC4 cells transfected with TACC2-FGFR2 or control plasmids to FGFR2 inhibitors, including BGJ398, AZD4547 and Erdafitinib. MKN45T: TACC2-FGFR2-expression MKN45 cells. NUGC4T:TACC2-FGFR2-expression NUGC4 cells 
$\mathbf{a}$
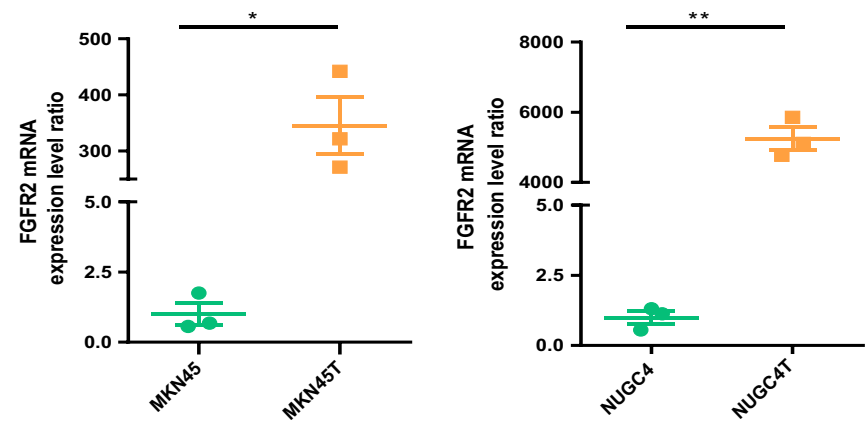

b

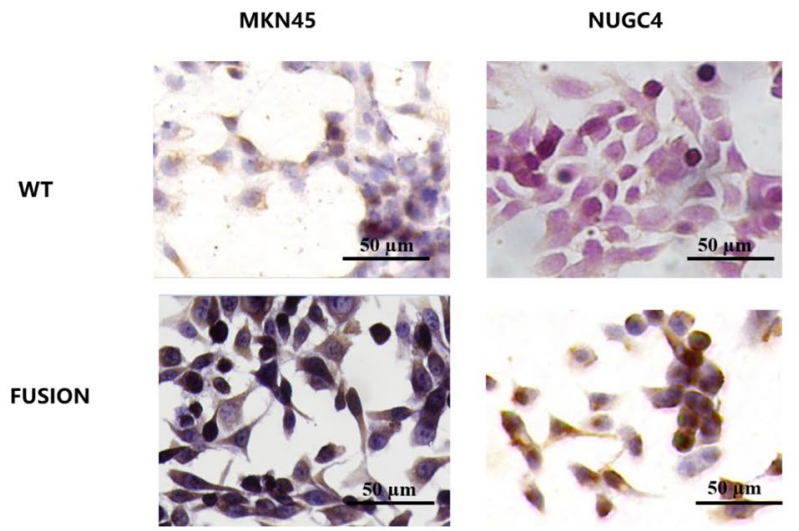

c

BGJ398

BGJ398
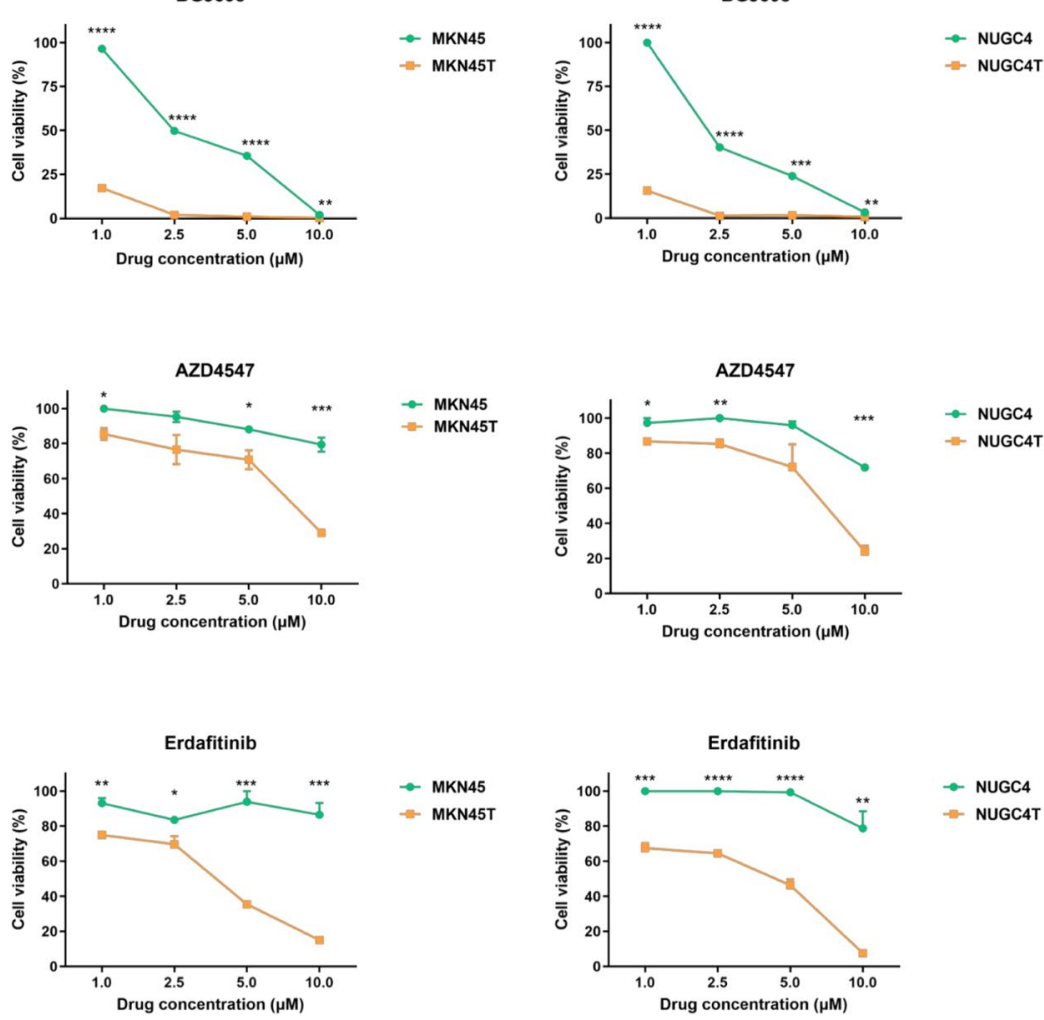

Fig. 4 (See legend on previous page.) 
prognosis [24]. FGFR2 inhibitors may be a good choice for PC GC with FGFR2 CNVs.

In addition, PC GC patients not only have more FGFR2 CNVs, but also have recurrent FGFR2 rearrangement (FGFR2/VTI1A and FGFR2/TACC2) in our study. FGFR rearrangements present in $13 \%$ to $17 \%$ of intrahepatic cholangiocarcinoma and represent driver mutations [26]. Several pre-clinical studies and clinical trials have demonstrated that FGFR2 rearrangements in cholangiocarcinoma can predict tumor sensitivity to FGFR2 inhibitors and become an important therapy in these highly selected patients [27-29]. To verify whether FGFR2 rearrangements can be a marker for targeted therapy in PC GC patients, we transfected TACC2-FGFR2 construct into GC cell lines (MKN45 and NUGC4), which has not been studied in PC GC before. We chose FGFR2 inhibitors, including AZD4547, Erdafitinib, and BGJ398 to treat GC cell lines with or without TACC2-FGFR2 fusion, and got similar results. Recently, many FGFR2 inhibitors are in the pipeline, these three drugs belonged to the first FGFR2 inhibitors studied. AZD4547 has been used in clinical trials of gastric adenocarcinoma [30], and Erdafitinib (Balversa ${ }^{\mathrm{TM}}$, Janssen Pharmaceutical Companies) [31] and BGJ398 (Infigratinib) [27, 32] was or will be approved by FDA for treatment of tumor with FGFR2 alterations. Our results firstly verified that the FGFR2 mRNA and protein expression level (Fig. 4b, c) were increased in GC cells with FGFR2 rearrangement and cells became more sensitive to FGFR2 inhibitors.

\section{Conclusion}

In conclusion, we firstly identified FGFR2 alteration was more frequently among PC GC than non-PC GC, including $\mathrm{CNVs}$ and rearrangements. Moreover, we verified TACC2-FGFR2 fusion could increase FGFR2 expression in mRNA and protein level, and GC cell lines with TACC2-FGFR2 fusion were more sensitive to FGFR2 inhibitors. All these results suggested that FGFR2 may be a potential therapeutic target for PC GC.

\section{Abbreviations}

AJCC: American Joint Committee on Cancer; CCK-8: Cell counting kit-8; CIN: Chromosomal instability; CNVs: Copy number variants; EBV: Epstein-Barr virus; FFPE: Formalin fixed paraffin embedded; FISH: Fluorescence in situ hybridization; GC: Gastric cancer; GEA: Gastroesophageal junction adenocarcinoma; GS: Genomically stable; IHC: Immunohistochemistry; Indels: Short and long insertions/deletions; MMR: Mismatch repair; MSI: Microsatellite instability; MSI$\mathrm{H}$ : Microsatellite instability-high; NGS: Next-generation sequencing; OS: Overall survival; PC: Poorly cohesive; qRT-PCR: Quantitative real time polymerase chain reaction; RNA-seq: RNA sequencing; SNVs: Single nucleotide variants; SV: Structural variants; TCGA: The Cancer Genome Atlas; TMB: Tumor mutation burden; TMB-H:Tumor mutational burden-high; TMB-L: Tumor mutational burden-low; TP53: Tumor protein 53.
}

\section{Supplementary Information}

The online version contains supplementary material available at https://doi. org/10.1186/s12967-021-03079-8.

Additional file 1: Figure S1. Schema diagram of NGS process. NGS next generation sequencing. Table S1. Univariate and multivariate analyses for PC GC patients. Figure S2. FISH detection of the FGFR2 fusion. Yellow arrows indicate separate location of different FGFR2 exons (red and green). Scale bar $=5 \mu \mathrm{m}$. Figure S3. RNA-sequence reads map of TACC2-FGFR.

\section{Acknowledgements}

The authors would like to thank all patients who participated in this study.

\section{Authors' contributions}

YW: Drafting the article; TS: Revising it critically for important intellectual content; XW: The acquisition of data; JH: Visualization; LY: Analysis and interpretation of data; QL: The acquisition of data; NW: Analysis and interpretation of data; BL: Supervision, final approval of the version to be submitted; JW: Conceptualization, funding acquisition. All authors read and approved the final manuscript.

\section{Funding}

This work was funded by Grants from National Natural Science Foundation of China (82073382) and the Distinguished Young Scholars of Jiangsu Province (BK20190001). The funding sources had no role in the study design, data collection, data analysis, data interpretation, or writing of this review. The funding sources have no involvement in study design; the collection, analysis and interpretation of data; in the writing of the report; or in the decision to submit the paper for publication.

\section{Availability of data and materials}

The data described in this manuscript are contained in published articles or available from the corresponding author upon reasonable request.

\section{Declarations}

Ethics approval and consent to participate

This study was conducted in accordance with the code of ethics of the World Medical Association (Declaration of Helsinki) and approved by the Ethics Committee of Nanjing Drum Tower Hospital (No. 2016-196-01).

\section{Consent for publication}

Not applicable.

\section{Competing interests}

The authors have no conflicts of interest to declare.

\section{Author details}

${ }^{1}$ The Comprehensive Cancer Centre of Nanjing Drum Tower Hospital, The Affiliated Hospital of Nanjing University Medical School \& Clinical Cancer Institute of Nanjing University, No. 321, Zhongshan Road, Nanjing 210008, China. ${ }^{2}$ The Comprehensive Cancer Centre of Nanjing Drum Tower Hospital, Clinical College of Nanjing Medical University, No. 321, Zhongshan Road, Nanjing 210008 , China. ${ }^{3}$ OrigiMed, No. 115, Xinjun Ring Road, Shanghai 201114, China.

Received: 10 May 2021 Accepted: 13 September 2021

Published online: 22 September 2021

\section{References}

1. Bray F, Ferlay J, Soerjomataram I, Siegel RL, Torre LA, Jemal A. Global cancer statistics 2018: GLOBOCAN estimates of incidence and mortality worldwide for 36 cancers in 185 countries. CA Cancer J Clin. 2018;68:394-424. 
2. Smyth EC, Nilsson M, Grabsch HI, van Grieken NCT, Lordick F. Gastric cancer. The Lancet. 2020;396:635-48.

3. Mariette C, Carneiro F, Grabsch HI, van der Post RS, Allum W, de Manzoni $\mathrm{G}$, et al. Consensus on the pathological definition and classification of poorly cohesive gastric carcinoma. Gastric Cancer. 2019;22:1-9.

4. Wagner AD, Grabsch HI, Mauer M, Marreaud S, Caballero C, ThussPatience P, et al. EORTC-1203-GITCG - the "INNOVATION"-trial: Effect of chemotherapy alone versus chemotherapy plus trastuzumab, versus chemotherapy plus trastuzumab plus pertuzumab, in the perioperative treatment of HER2 positive, gastric and gastroesophageal junction adenocarcinoma on pathologic response rate: a randomized phase IIintergroup trial of the EORTC-Gastrointestinal Tract Cancer Group, Korean Cancer Study Group and Dutch Upper Gl-Cancer group. BMC Cancer. 2019;19:494.

5. Sarriugarte Lasarte A, Garcla Alberdi E, Martinez Indart L, Gutierrez Grijalba O, Albarez Abad I, Guerra Lerma M, et al. "From Lauren's diffuse gastric cancer to WHO's poorly cohesive carcinoma." Clinicopathological and prognostic characteristics. Rev Esp Enferm Dig. 2020;113:324-32.

6. Tan IB, Ivanova T, Lim KH, Ong CW, Deng N, Lee J, et al. Intrinsic subtypes of gastric cancer, based on gene expression pattern, predict survival and respond differently to chemotherapy. Gastroenterology. 2011;141:47685,85 e1-11.

7. Lei Z, Tan IB, Das K, Deng N, Zouridis H, Pattison S, et al. Identification of molecular subtypes of gastric cancer with different responses to PI3kinase inhibitors and 5-fluorouracil. Gastroenterology. 2013;145:554-65.

8. Cancer Genome Atlas Research N. Comprehensive molecular characterization of gastric adenocarcinoma. Nature. 2014;513:202-9.

9. Cristescu R, Lee J, Nebozhyn M, Kim KM, Ting JC, Wong SS, et al. Molecular analysis of gastric cancer identifies subtypes associated with distinct clinical outcomes. Nat Med. 2015;21:449-56.

10. Alborelli I, Leonards K, Rothschild SI, Leuenberger LP, Savic Prince S, Mertz KD, et al. Tumor mutational burden assessed by targeted NGS predicts clinical benefit from immune checkpoint inhibitors in non-small cell lung cancer. J Pathol. 2020;250:19-29.

11. Tappeiner E, Finotello F, Charoentong P, Mayer C, Rieder D, Trajanoski Z. TIminer: NGS data mining pipeline for cancer immunology and immunotherapy. Bioinformatics. 2017;33:3140-1.

12. Rosenthal R, McGranahan N, Herrero J, Taylor BS, Swanton C. DeconstructSigs: delineating mutational processes in single tumors distinguishes DNA repair deficiencies and patterns of carcinoma evolution. Genome Biol. 2016;17:31.

13. Huang $\mathrm{KH}$, Chen $\mathrm{MH}$, Fang $\mathrm{WL}$, Lin $\mathrm{CH}$, Chao Y, Lo SS, et al. The clinicopathological characteristics and genetic alterations of signet-ring cell carcinoma in gastric cancer. Cancers (Basel). 2020;12:2318.

14. Lee D, Ham IH, Son SY, Han SU, Kim YB, Hur H. Intratumor stromal proportion predicts aggressive phenotype of gastric signet ring cell carcinomas. Gastric Cancer. 2017;20:591-601.

15. Shu Y, Zhang W, Hou Q, Zhao L, Zhang S, Zhou J, et al. Prognostic significance of frequent CLDN18-ARHGAP26/6 fusion in gastric signet-ring cell cancer. Nat Commun. 2018;9:2447.

16. Xu B, Liu F, Liu Q, Shi T, Wang Z, Wu N, et al. Highly expressed Claudin18.2 as a potential therapeutic target in advanced gastric signet-ring cell carcinoma (SRCC). J Gastrointest Oncol. 2020;11:1431-9.

17. Wang K, Yuen ST, Xu J, Lee SP, Yan HH, Shi ST, et al. Whole-genome sequencing and comprehensive molecular profiling identify new driver mutations in gastric cancer. Nat Genet. 2014;46:573-82.

18. Kwon CH, Kim YK, Lee S, Kim A, Park HJ, Choi Y, et al. Gastric poorly cohesive carcinoma: a correlative study of mutational signatures and prognostic significance based on histopathological subtypes. Histopathology. 2018;72:556-68.

19. Yang L, Luquette LJ, Gehlenborg N, Xi R, Haseley PS, Hsieh CH, et al. Diverse mechanisms of somatic structural variations in human cancer genomes. Cell. 2013;153:919-29.

20. Turner N, Grose R. Fibroblast growth factor signalling: from development to cancer. Nat Rev Cancer. 2010;10:116-29.

21. Ahn S, Lee J, Hong M, Kim ST, Park SH, Choi MG, et al. FGFR2 in gastric cancer: protein overexpression predicts gene amplification and high H-index predicts poor survival. Mod Pathol. 2016;29:1095-103.

22. Kim HS, Kim JH, Jang HJ, Han B, Zang DY. Pathological and prognostic impacts of FGFR2 overexpression in gastric cancer: a meta-analysis. J Cancer. 2019;10:20-7.

23. Hattori $Y$, Itoh H, Uchino S, Hosokawa K, Ochiai A, Ino Y, et al. Immunohistochemical detection of K-sam protein in stomach cancer. Clin Cancer Res. 1996;2:1373-81.

24. Wainberg ZA, Enzinger PC, Kang Y-K, Yamaguchi K, Qin S, Lee K-W, Oh SC, Li J, Turk HM. A double-blind randomized study of bemarituzumab (bema) plus mFOLFOX6 versus placebo plus mFOLFOX6 as first-line treatment for advanced gastric/gastroesophageal junction cancer (FIGHT). In: ASCO gastrointestinal cancer symposium. 2021;LBA160.

25. Catenacci DVT, Rasco D, Lee J, Rha SY, Lee K-W, Bang YJ, Bendell J, Enzinger P, Marina N, Xiang H, Deng W, Powers J, Wainberg ZA. Phase I escalation and expansion study of bemarituzumab (FPA144) in patients with advanced solid tumors and FGFR2b-selected gastroesophageal adenocarcinoma. J Clin Oncol. 2020;38:2418-26.

26. Graham RP, Barr Fritcher EG, Pestova E, Schulz J, Sitailo LA, Vasmatzis G, et al. Fibroblast growth factor receptor 2 translocations in intrahepatic cholangiocarcinoma. Hum Pathol. 2014:45:1630-8.

27. Javle M, Lowery M, Shroff RT, Weiss KH, Springfeld C, Borad MJ, et al. Phase II study of BGJ398 in patients with FGFR-altered advanced cholangiocarcinoma. J Clin Oncol. 2018;36:276-82.

28. Ross JS, Wang K, Gay L, Al-Rohil R, Rand JV, Jones DM, et al. New routes to targeted therapy of intrahepatic cholangiocarcinomas revealed by nextgeneration sequencing. Oncologist. 2014;19:235-42.

29. Farshidfar F, Zheng S, Gingras MC, Newton $Y$, Shih J, Robertson AG, et al. Integrative genomic analysis of cholangiocarcinoma identifies distinct IDH-mutant molecular profiles. Cell Rep. 2017;19:2878-80.

30. Van Cutsem E, Bang YJ, Mansoor W, Petty RD, Chao Y, Cunningham $\mathrm{D}$, et al. A randomized, open-label study of the efficacy and safety of AZD4547 monotherapy versus paclitaxel for the treatment of advanced gastric adenocarcinoma with FGFR2 polysomy or gene amplification. Ann Oncol. 2017;28:1316-24.

31. Loriot $Y$, Necchi A, Park SH, Garcia-Donas J, Huddart R, Burgess E, et al. Erdafitinib in locally advanced or metastatic urothelial carcinoma. N Engl J Med. 2019;381:338-48.

32. Nogova L, Sequist LV, Perez Garcia JM, Andre F, Delord JP, Hidalgo M, et al. Evaluation of BGJ398, a fibroblast growth factor receptor 1-3 kinase inhibitor, in patients with advanced solid tumors harboring genetic alterations in fibroblast growth factor receptors: results of a global phase I, dose-escalation and dose-expansion study. J Clin Oncol. 2017;35:157-65.

\section{Publisher's Note}

Springer Nature remains neutral with regard to jurisdictional claims in published maps and institutional affiliations. 